\title{
Control of high-frequency AC link electronic transformer
}

\author{
H. Krishnaswami and V. Ramanarayanan
}

\begin{abstract}
An isolated high-frequency link AC/AC converter is termed an electronic transformer. The electronic transformer has size and cost advantages over a conventional transformer because of high-frequency operation of the magnetic core. Of the various topologies of electronic transformer, the high-frequency AC link electronic transformer achieves high-frequency AC power transformation without a DC link. The circuit uses the standard H-bridge, one on either side of the high-frequency transformer. A novel PWM scheme is proposed, which symmetrically delays and advances the phase of the left and right legs of the front-side converter with respect to the outputside converter. The proposed scheme introduces freewheeling sub-periods, which results in zero voltage switching in the output-side converter. The electronic transformer as an AC automatic voltage regulator (AVR) offers distinct advantages over a conventional servo voltage stabiliser in terms of size and speed of response. The AVR application is discussed and experimental results of a $500 \mathrm{VA}$ AVR are presented. A four-quadrant switch presents difficulties in turning off inductive load current because of the absence of a freewheeling path in the switch. A biasing circuit is proposed to convert the potentially lossy switching transition into lossless transition. Simulation and experimental results with the biasing circuit are presented.
\end{abstract}

\section{Introduction}

Line frequency transformers $(50$ and $60 \mathrm{~Hz})$ are heavy and bulky items in power conversion and distribution systems. Transformer size is inversely proportional to the frequency of operation and saturation flux density. Hence a reduction in volume and weight can be obtained by high-frequency operation of the magnetic core. Introduction of a highfrequency link to realise a small-size electronic transformer has been widely discussed in the literature [1-4]. Low cost and easy availability of ferrite core material has helped in the implementation of high-frequency link power transformation.

The electronic transformer utilises power electronic converters along with a high-frequency transformer to obtain overall size and cost advantages over a conventional transformer. Several topologies of electronic transformer have been discussed in [4]. One of the topologies of the electronic transformer is the high-frequency AC link $\mathrm{AC} /$ $\mathrm{AC}$ converter first proposed in [1]. It has two cycloconverters with a high-frequency AC link in between, as shown in Fig. 1. H-bridge and push-pull circuits have been proposed in [2] and [3], respectively. It may be seen that the literature has extended the application of high-frequency link AC transformation not only for low-level electronic power

Paper first received 9th May and in final form 19th August 2004. Originally published online: 8th April 2005

H. Krishnaswami was with the Department of Electrical Engineering, Indian Institute of Science, India, and is now with the GE Healthcare Technologies, 1584 E-Block, AECS Layout, Kundalahalli, Bangalore, Karnataka 560037, India

V. Ramanarayanan is with the Department of Electrical Engineering, Indian Institute of Science, Bangalore, India

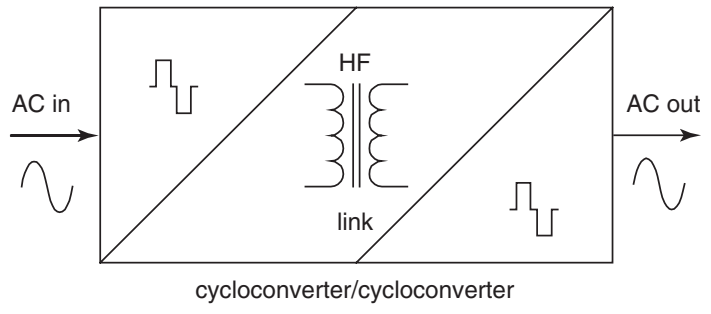

Fig. 1 Electronic transformer using HF AC link

conversion but also for medium-level distribution power transformers $[2,4]$. At such high powers the authors have proposed only voltage transformation and isolation. The major payoff expected is size reduction. The advantages extend to more innovative control possibilities as well.

\section{Principle of operation}

The principle of operation of the high-frequency AC link electronic transformer is based on amplitude modulation. Consider a low-frequency sine wave $(50 \mathrm{~Hz}) v_{\text {in }}(t)$ being modulated by a high-frequency square wave. These two are defined as:

$$
\begin{aligned}
& v_{\text {in }}(t)=V_{\text {in }} \sin \left(w_{i} t\right) \\
& H_{M}(t)=\left\{\begin{array}{cc}
1 & 0 \leq t \leq T_{s} / 2 \\
-1 & T_{s} / 2 \leq t \leq T_{s}
\end{array} T_{s}=\frac{2 \pi}{w_{s}}\right\}
\end{aligned}
$$

The resultant voltage after modulation can be obtained by multiplying $v_{\text {in }}(t)$ and $H_{M}(t)$, the Fourier series of which is given by,

$$
\begin{aligned}
v_{H F}(t)= & \frac{2 V_{i n}}{\pi} \sum_{n=1}^{\infty} \frac{1}{(2 n-1)}\left[\cos \left((2 n-1) w_{s}-w_{i} t\right)\right. \\
& \left.-\cos \left((2 n-1) w_{s}+w_{i} t\right)\right]
\end{aligned}
$$




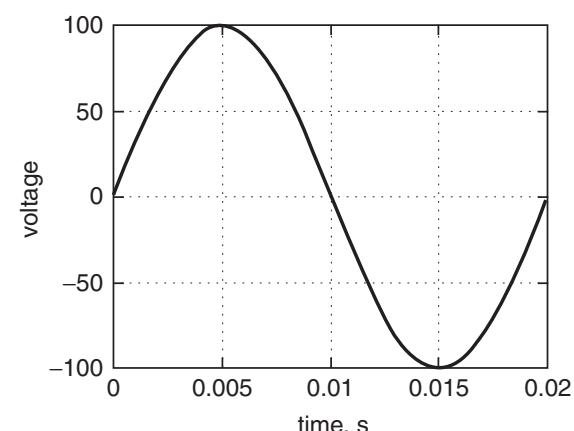

a

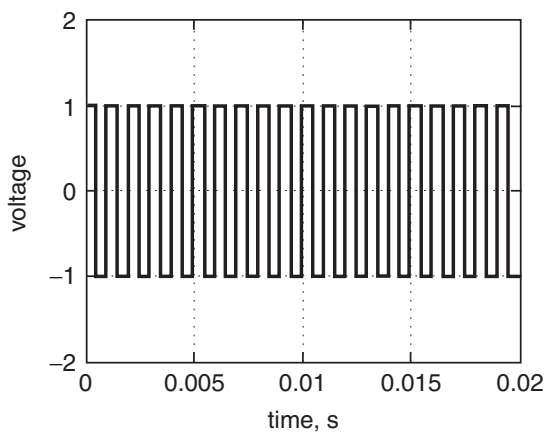

b

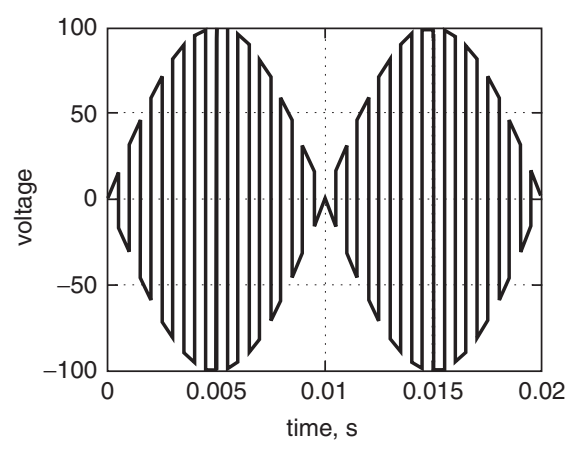

$c$

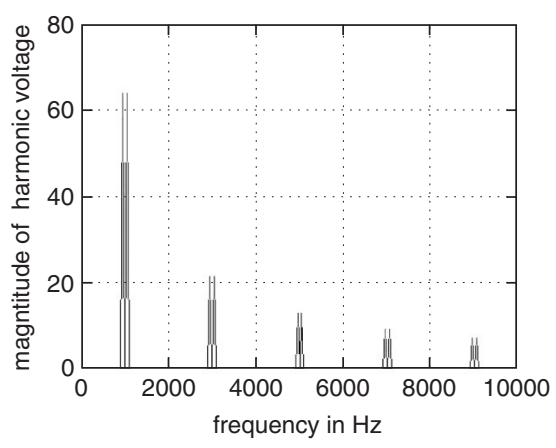

d

Fig. 2 Waveforms illustrating derivation of $v_{H F}(t)$

a $v_{\text {in }}(t)$

$b H_{M}(t)$

$c v_{H F}(t)$

$d$ Fourier spectrum of $v_{H F}(t)$

Equation (2) proves that the lowest harmonic starts at $w_{s} \pm w_{i}$. Hence this voltage can be transformed with a high-frequency transformer. The modulated waveform is shown in Fig. 2.

To control the AC output voltage [2] has proposed the demodulating function $H_{D}(t)$ as a delayed signal of $H_{M}(t)$, which is defined as:

$$
H_{D}(t)=\left\{\begin{array}{ll}
1 & \alpha \leq t \leq \frac{T_{s}}{2}+\alpha \\
-1 & \frac{T_{s}}{2}+\alpha \leq t \leq T_{s}+\alpha
\end{array}\right\} 0 \leq \alpha \leq \frac{T_{s}}{2}
$$

Evaluating the Fourier series of the output voltage, the fundamental output voltage $V_{\text {fund }}$ as a function of $\alpha$ is obtained as:

$$
\begin{aligned}
V_{\text {fund }}=V_{\text {in }}\left(1-\frac{4 \alpha}{T_{s}}\right) \quad \text { when } & \alpha=0, & V_{\text {fund }}=V_{\text {in }} \\
& \alpha=T_{s} / 2, & V_{\text {fund }}=-V_{\text {in }} \\
& \alpha=T_{s} / 2, & V_{\text {fund }}=0
\end{aligned}
$$

This paper presents the standard $\mathrm{H}$-bridge adapted to $\mathrm{AC}$ AC power conversion. The circuit is illustrated in Fig. 3. When the above-mentioned control is applied i.e., $H_{M}(t)$ controlling converter 1 and $\mathrm{H}_{\mathrm{D}}(\mathrm{t})$ controlling converter 2 , the unfiltered output voltage obtained is as shown in Fig. 4 along with the frequency spectrum. In Fig. 4, it is observed that with the above scheme the output voltage waveform is bipolar. Also since the transformer voltage does not have any zero voltage instants, i.e. no freewheeling sub-periods, some of the switching transitions in the converters are hard switching transitions. In this paper, an alternative control method is proposed wherein some of the switches achieve soft transitions.

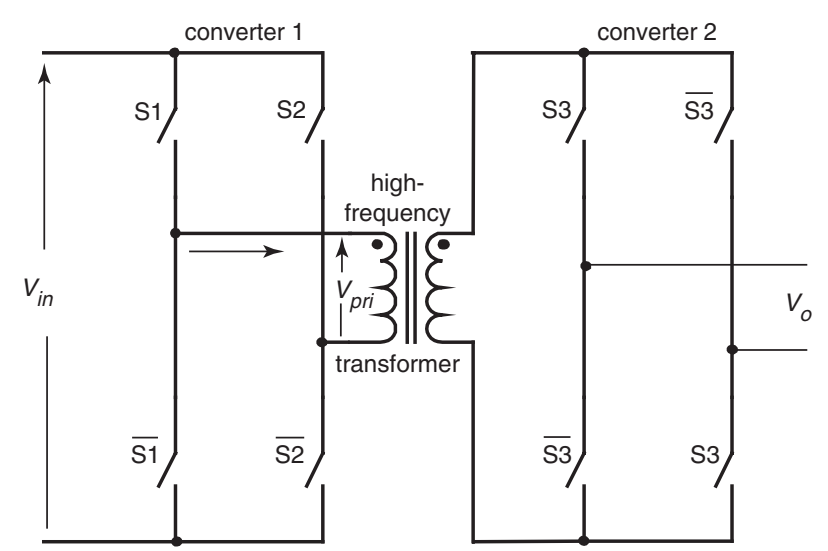

Fig. 3 Topology of high-frequency AC link electronic transformer

\section{Proposed symmetric modulation scheme}

In this paper, a symmetric modulation scheme is proposed wherein $H_{M}(t)$ and $H_{D}(t)$ are defined as:

$$
\begin{gathered}
H_{M}(t)=\left\{\begin{array}{ll}
1 & \alpha \leq t \leq T_{s} / 2 \\
-1 & T_{s} / 2+\alpha \leq t \leq T \\
0 & \text { elsewhere }
\end{array}\right\} \\
H_{D}(t)=\left\{\begin{array}{cl}
1 & 0 \leq t \leq T_{s} / 2 \\
-1 & T_{s} / 2 \leq t \leq T_{s}
\end{array}\right\}
\end{gathered}
$$

$H_{M}(t)$ controls the switches in converter 1 in Fig. 3. $H_{D}(t)$ controls the switches in converter 2. Evaluating the Fourier series of the unfiltered output voltage, the fundamental voltage $V_{\text {fund }}$ as a function of $\alpha$ is obtained as:

$$
\begin{array}{rlrl}
V_{\text {fund }}=V_{\text {in }}\left(1-\frac{2 \alpha}{T_{s}}\right) \quad \text { when } & \alpha=0, & & V_{\text {fund }}=V_{\text {in }} \\
& \alpha=T_{s} / 2, & V_{\text {fund }}=0 \\
& \alpha=T_{s}, & & V_{\text {fund }}=-V_{\text {in }}
\end{array}
$$

The nature of the function $H_{M}(t)$ implies that converter 1 is phase modulated. The control pulses for switches S1-S3 derived from $H_{M}(t)$ and $H_{D}(t)$ are shown in Fig. 5. It is clear from (6) that, when $\alpha$ is varied from 0 to $T_{s}$, the output voltage varies from $V_{\text {in }}$ to $-V_{\text {in }}$ through zero. Figure 6 shows the unfiltered output voltage waveforms for the 

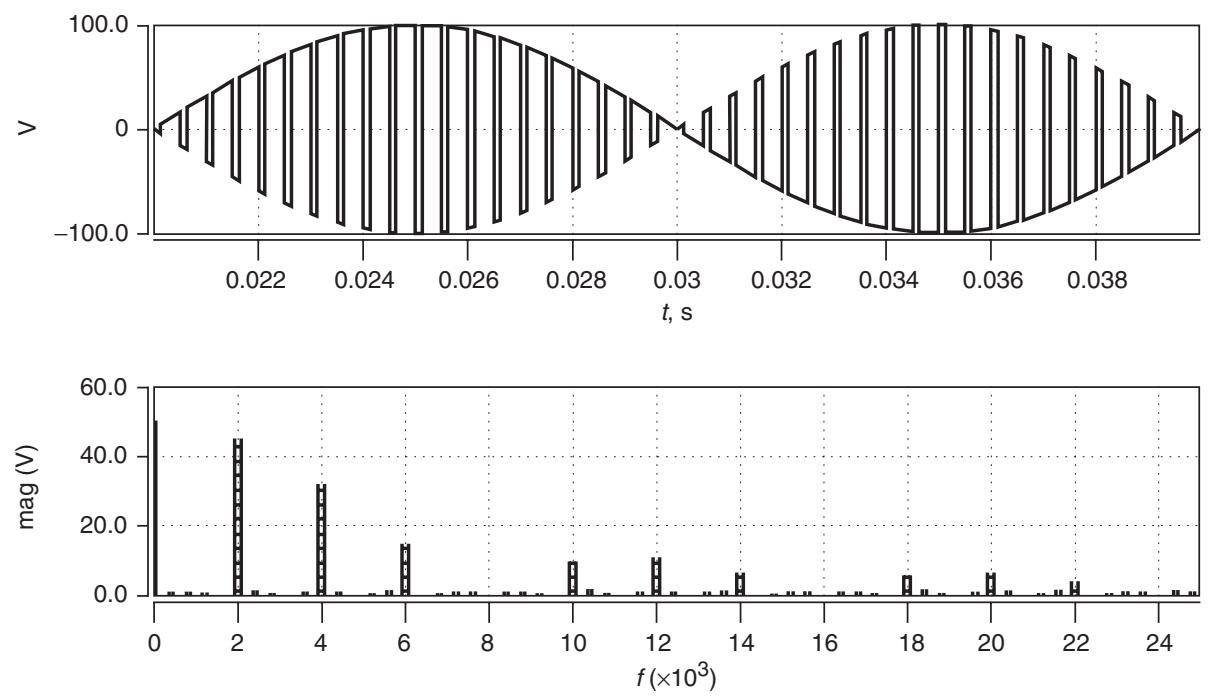

Fig. 4 Unfiltered output voltage waveform for existing control scheme

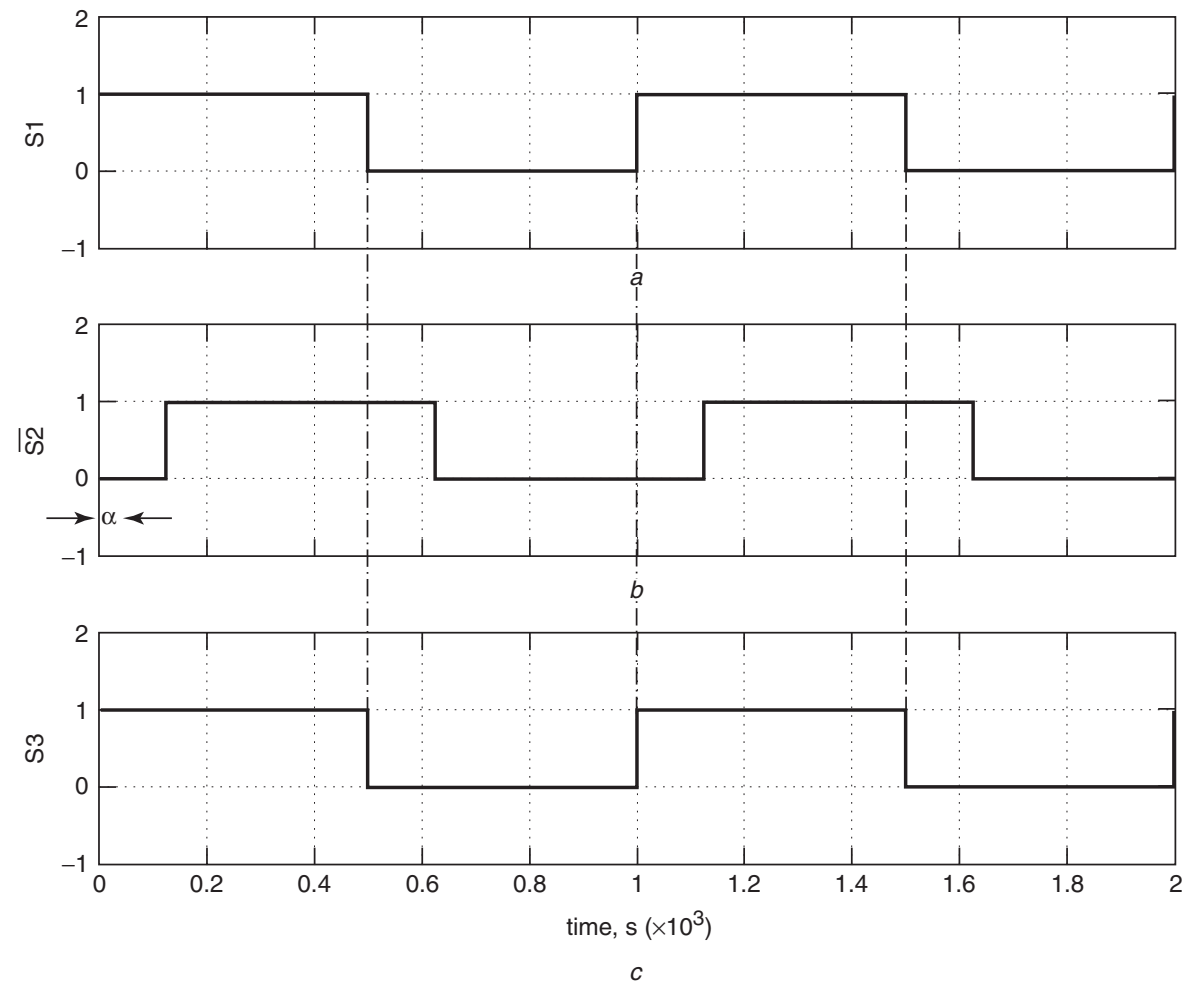

Fig. 5 Control signals for switches $S 1-S 3$ derived from $H_{M}(t)$ and $H_{D}(t)$

proposed control scheme. Note the difference in waveform in Figs. 4 and 6.

At $t=T_{S} / 2$ in Fig. 5, switches $\mathrm{S} 1$ in converter 1 and $\mathrm{S} 3$ in converter 2 change from +1 to 0 simultaneously. If $\alpha$ in Fig. 5 is symmetrically distributed on either side of $T_{s} / 4$ within the half cycle $T_{S} / 2$ then, at any point of time only one transition takes place, i.e. either in converter 1 or in converter 2. Earlier S2 was phase shifted with respect to S1. After this modification, the phase variations of S1 and S2 with respect to $\mathrm{S} 3$ are positive and negative, respectively. Hence it is given the name symmetric modulation. The resultant pulses for the switches are shown in Fig. 7. This symmetric nature of the scheme proves advantageous during the switching process of converter 2 .

AC/AC converters employing four quadrant switches have the problem of high-current and high-voltage surges during commutation. This decreases the efficiency of the converter as well as the reliability of the system. These facts are a great barrier to making this converter practicable. In this proposed control scheme the transformer voltage waveform has zero voltage states. Owing to the symmetric nature of the scheme all the switches in converter 2 are turned on at this instant. Hence zero voltage switching (ZVS) is achieved in converter 2. A method to reduce switching losses in converter 1 is discussed in Section 5 of this paper. Also the control scheme naturally leads to unipolar output voltage, which has its advantages known in the literature.

Two methods of realisation of this PWM scheme are proposed in this paper. The first method uses a triangular wave and can give only an in-phase output voltage, i.e. $\alpha$ can vary from 0 to $T_{s} / 2$ only. The second method uses a 

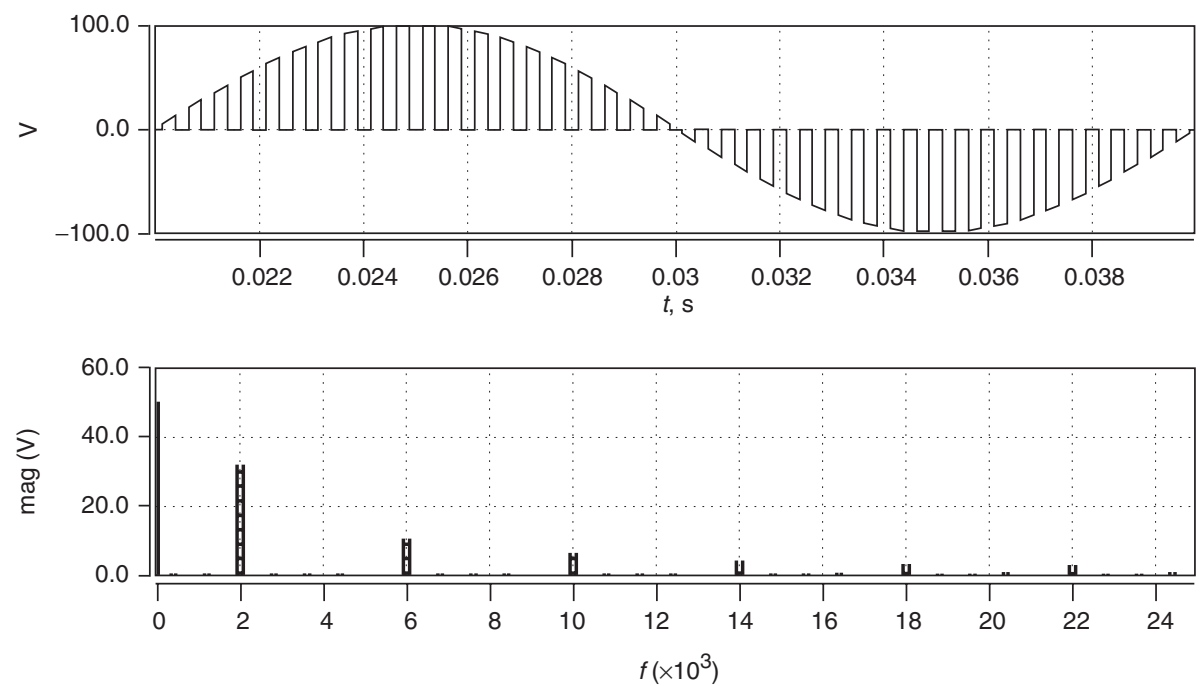

Fig. 6 Unfiltered output waveform for proposed control scheme
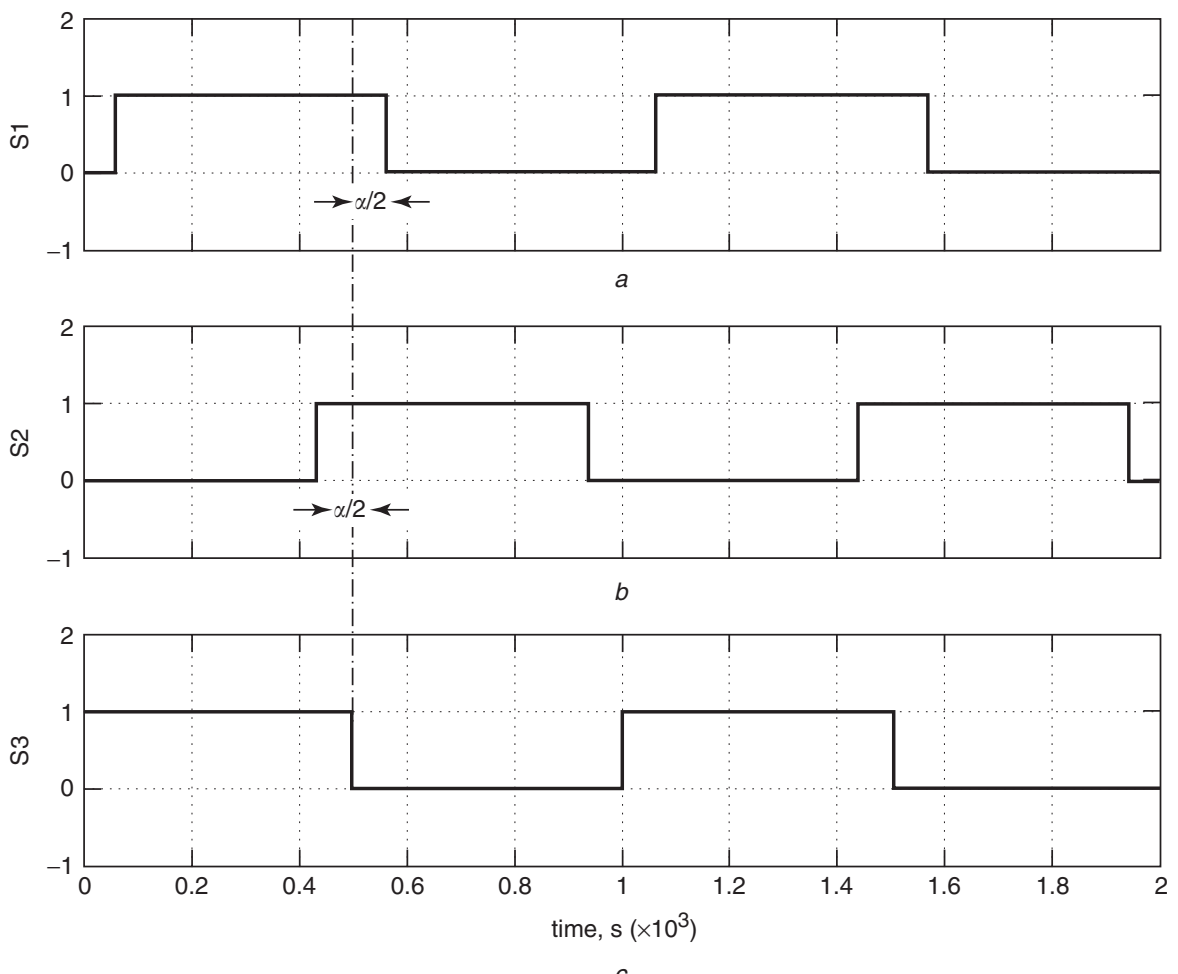

Fig. 7 Control signals for switches S1-S3 to illustrate symmetric modulation

double ramp carrier wave to give both in-phase and out-ofphase output voltage. Figs. $8 a$ and $b$ illustrate the two methods. In Fig. $8 b V_{R}$ is the carrier voltage and $V_{C}$ is the control voltage. $V_{R}$ has two identical ramps shifted by $180^{\circ}$. This method does not introduce a discontinuity at the zero output voltage point, i.e. when $\alpha=T_{s} / 2$, hence it is useful in closed-loop control. The second method finds its application in control of the automatic voltage regulator (AVR), which is explained in the following Section.

\section{Application as an automatic voltage regulator}

Power-line disturbances such as undervoltage, overvoltage, voltage sags and swells in sensitive equipment such as computers, communication services etc., can often lead to loss of valuable data and interruption of communication services. Traditionally servo voltage stabilisers fill this need. They use a variable autotransformer and an isolation transformer to inject a compensating voltage in series with the utility power. Slow dynamic response and bulky line frequency transformer are the main disadvantages of such a system. Various methods of providing variable AC have been discussed in $[5,6]$. But the line frequency magnetics still form a part of the system. An electronic transformer can replace the line frequency autotransformer and isolation transformer by a single high-frequency transformer. The size of the system reduces drastically as a result. Such an AVR is shown in Fig. 9. The output voltage of the AVR can be derived as:

$$
V_{o}=(1-n+2 D n) \times V_{\text {in }} \text { where } D=\frac{\alpha}{T_{S}}
$$




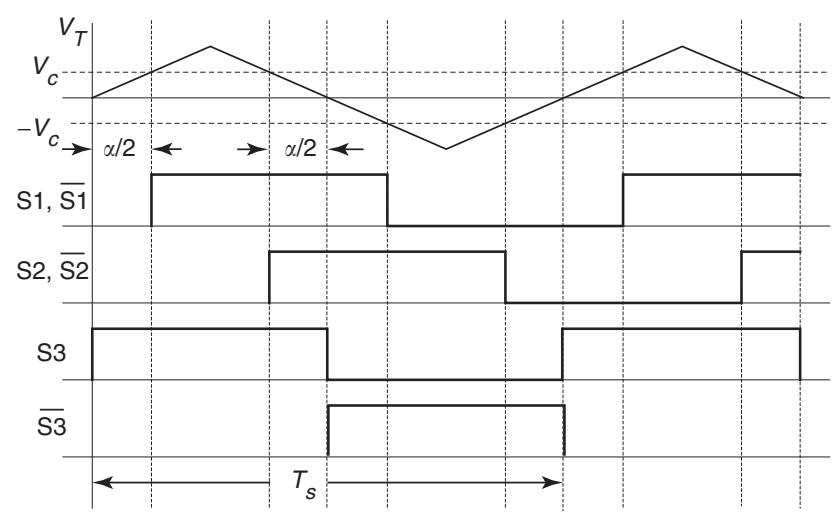

a

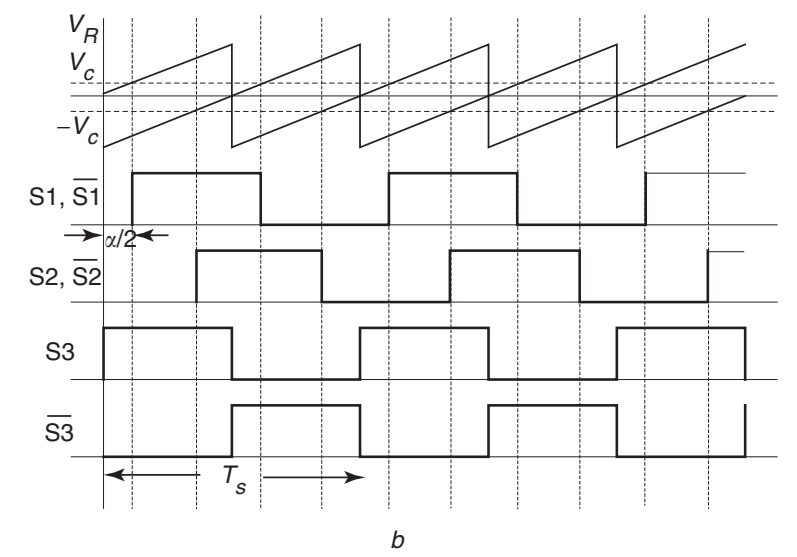

Fig. 8 Control scheme realisations $a$ Triangular carrier $b$ Double ramp carrier

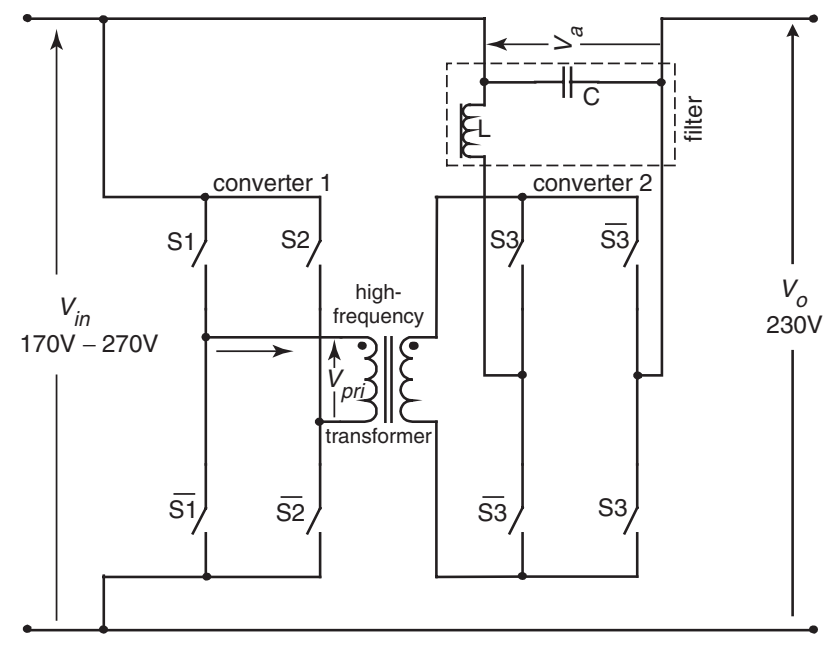

Fig. 9 AVR using high-frequency AC link electronic transformer

where $V_{o}$ is the regulated output voltage of the AVR and $n$ is the transformer turns ratio. Thus when

$$
\begin{aligned}
& D=0, V_{o}=(1-n) V_{\text {in }} \text { (buck) } \\
& D=0.5, V_{o}=V_{\text {in }} \\
& \left.D=1, V_{o}=(1+n) V_{\text {in }} \text { (boost }\right)
\end{aligned}
$$

\section{$5 \quad$ Biasing circuit}

In this Section a biasing circuit is proposed to reduce the switching losses of converter 1 . Converter 2 switching transitions are already ZVS, as explained in Section 3. Consider the operation of a single leg in converter 1 , as shown in Fig. 2. A capacitive snubber is added across each switch to prevent the interruption of load current. This capacitance may be the device capacitance or an external capacitance added across the switch. To prevent the source getting short-circuited, a dead time is given between two switches in one leg. Figure 10 explains the working of the single-leg circuit during the dead time with the transition from $\overline{\mathrm{S} 1}$ to $\mathrm{S} 1$ taken for example. Both positive and negative input voltages are considered with the current in the same direction.
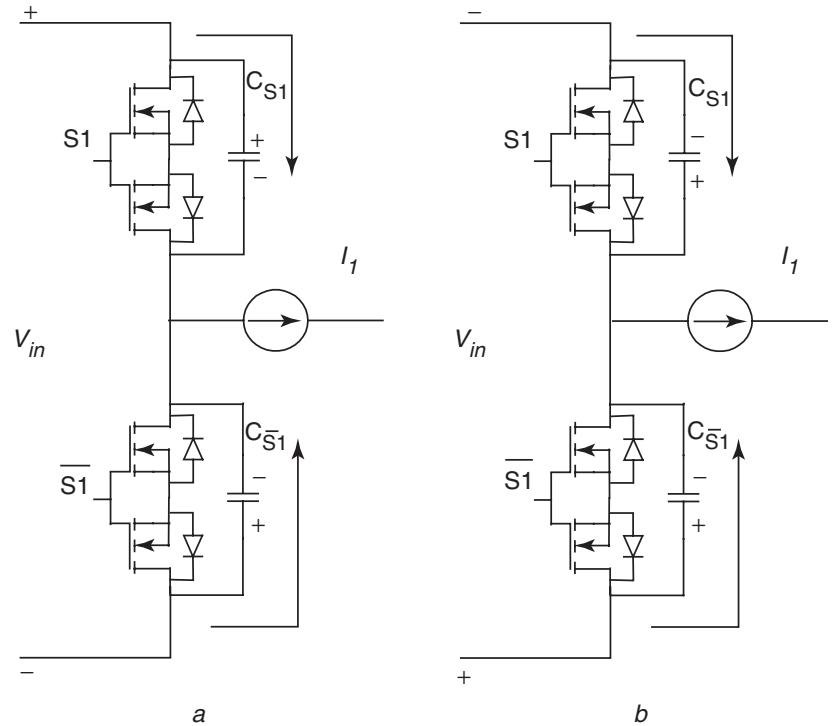

Fig. 10 Switching transition from $\overline{\mathrm{S} 1}$ to $S 1$ during delay time showing direction of current and charging of capacitors $C_{S I}$ and $\mathrm{C}_{\overline{\mathrm{S} 1}}$ $a$ Lossy transition

$b$ Lossless transition

Consider initially that $\overline{\mathrm{S} 1}$ is on and the load current is flowing through $\overline{\mathrm{S} 1}$, as shown in Fig. $10 a$. When $\overline{\mathrm{S} 1}$ is turned off, the inductive load current splits between the two capacitors, $\mathrm{C}_{\mathrm{S} 1}$ and $\mathrm{C}_{\overline{\mathrm{S} 1}}$. The load current is not in the proper direction to aid the charge transfer from $\mathrm{C}_{\mathrm{S} 1}$ and $\mathrm{C}_{\overline{\mathrm{S} 1}}$. Instead, the capacitance across switch $\mathrm{S} 1$ gets charged continuously to a value of higher than the bus voltage. Both $\mathrm{C}_{\mathrm{S} 1}$ and $\mathrm{C}_{\overline{\mathrm{S} 1}}$ are charged in the opposite direction, as in Fig. 10a. When S1 is now turned on, the stored energy gets dissipated in the device. This results in high current spikes. This is a lossy transition. Consider the operation in Fig. $10 b$. When $\overline{\mathrm{S} 1}$ is turned off, the reflected load current aids in the energy transfer between the output capacitance of the two complementary switches, $\mathrm{S} 1$ and $\overline{\mathrm{S} 1}$. When $\mathrm{C}_{\mathrm{S} 1}$ is discharged completely to zero, S1 can be turned on. This is a lossless transition. A method to prevent overvoltages has been proposed in [8], which controls each two-quadrant switch in a four-quadrant switch element. In this paper a novel biasing circuit is proposed to convert the potentially lossy transitions into lossless transitions.

Turn-on and turn-off losses are the two main constituents of switching losses. In ZVS converters, the device voltage is brought to zero just prior to turn-on. During turn-off the rate of voltage rise is limited, so that the device current falls to zero before the voltage rises substantially. In the electronic transformer circuit, the turn-off losses are negligible as a capacitive snubber is added across the switches. But during turn-on there exists a voltage across 
the MOSFET and the turn-on losses are determined by the energy stored in the capacitance across the MOSFETS (intrinsic and extrinsic). An analysis of converter 1 in Fig. 2 was performed to identify the lossy and lossless switching transitions (turn-on) in a manner similar to that shown in Fig. 10. Table 1 presents the results of the quadrant-byquadrant analysis of switching transitions in converter 1 . The quadrants are defined based on the fundamental load voltage and load current. Out of the 16 switching transitions in converter 1 eight are lossy and eight are lossless. It is observed that lossy and lossless transition depends on the quadrant of operation. Reference [9] has suggested an external biasing circuit to reduce the load dependence of the ZVS characteristics in a phase-modulated DC/DC converter. This technique of biasing appropriate legs with suitable currents to ensure ZVS is applied to the electronic transformer in this paper.

Table 1: Summary of switching transient analysis

\begin{tabular}{|c|c|c|c|c|}
\hline Switch & $\begin{array}{l}\text { Quadrant } \\
1\end{array}$ & $\begin{array}{l}\text { Quadrant } \\
2\end{array}$ & $\begin{array}{l}\text { Quadrant } \\
3\end{array}$ & $\begin{array}{l}\text { Quadrant } \\
4\end{array}$ \\
\hline $\mathrm{S} 1, \mathrm{~S}^{\prime}$ & lossy & lossless & lossy & lossless \\
\hline $\mathrm{S} 2, \mathrm{~S} 2^{\prime}$ & lossless & lossy & lossless & lossy \\
\hline
\end{tabular}

The basic principle of operation of the biasing circuit is that at the instant of switching transition the current is made lagging. The concept is illustrated in Fig. 11. An inductor is used across the midpoint of the split capacitor

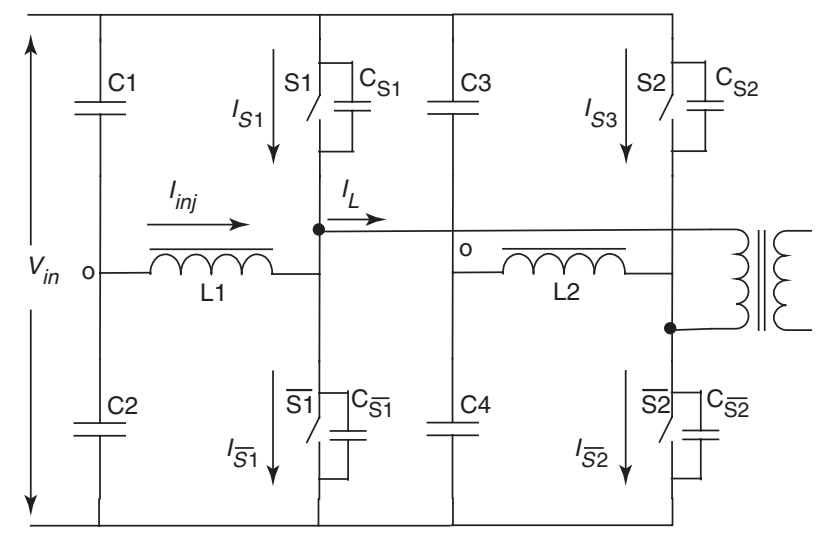

a

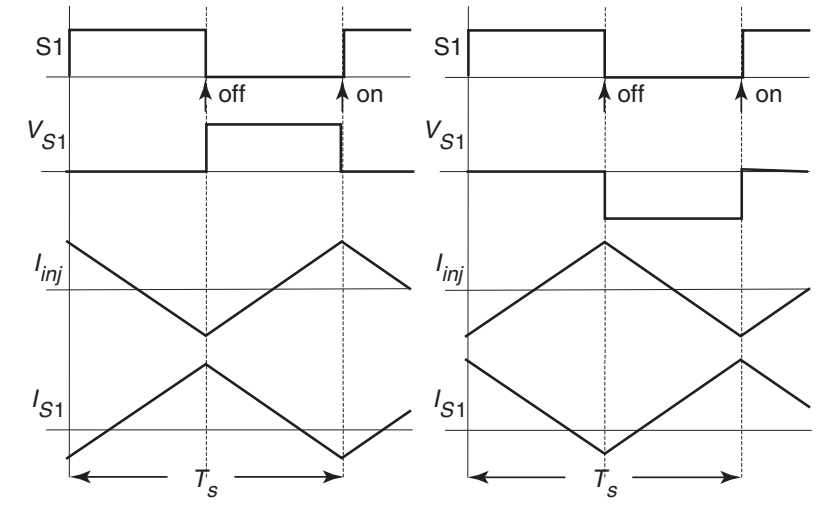

$b$

Fig. 11

$a$ Biasing circuit

$b$ Operation of biasing circuit explained with S1 turn-on (point ' $\mathrm{o}$ ') and the centre point of one leg. This biasing circuit injects high-frequency current into the system to overcome the effect of load current during transitions. The injected current is designed to be twice the value of peak of the reflected load current. The operation of the biasing circuit is explained considering the switching transitions from $\overline{\mathrm{S} 1}$ to $\mathrm{S} 1$. Before $\overline{\mathrm{S} 1}$ is turned off, it is carrying the sum of the reflected load current $\left(I_{\mathrm{L}}\right)$ and the injected current $\left(I_{i n j}\right)$ and the resultant switch current $\left(I_{\overline{\mathrm{S}}}\right)$ is positive (direction of $I_{\overline{\mathrm{S} 1}}$ shown in Fig. 13). Note that the same current $I_{\overline{\mathrm{Sl}}}$ is negative in a lossy transition. During the dead time, the injected current divides equally between the two capacitors $\mathrm{C}_{\mathrm{S} 1}$ and $\mathrm{C}_{\overline{\mathrm{S} 1}}$. Since $I_{\overline{\mathrm{S}} 1}$ is positive the capacitor $\mathrm{C}_{\mathrm{S} 1}$ gets discharged and the switch $\mathrm{S} 1$ is turned on when the voltage across it is zero. In this way ZVS is achieved in S1 in quadrant 1. A similar analysis is carried out for all switches in converter 1 and it is found that the biasing circuit ensures ZVS for switches S1 and S2 in all four quadrants.

\section{Experimental results}

A 500 VA prototype has been fabricated to demonstrate the suitability of the electronic transformer as an AVR and to demonstrate the advantages of the proposed control scheme. The input voltage range is $170-270 \mathrm{~V}$. The output voltage is regulated to $230 \mathrm{~V}$ and with a $500 \mathrm{~W}$ load the load current is $2.17 \mathrm{~A}$. The switching frequency of the power devices is fixed at $20 \mathrm{kHz}$. The voltage is added or subtracted in series with the mains (series voltage regulator) and hence the high-frequency transformer handles only a certain fraction of the output power. The maximum voltage added is $60 \mathrm{~V}$ and hence the high-frequency transformer VA rating is $230 \mathrm{~V} \times 2.17 \mathrm{~A}$, which is equal to $130 \mathrm{VA}$. In the developed AVR the transformer is designed to handle a maximum power of 175VA. The core area of the transformer was $235 \mathrm{~mm}^{2}$, which is lesser than a conventional line frequency transformer handling the same amount of power. The power MOSFETS used were IRF840, which has an on-state resistance of $0.85 \Omega$.

A major part in the control circuit is the generation of double ramp. Digital circuitry with counters and comparators has been used to generate the ramps. The MOSFETS were driven through pulse transformers to provide isolation between the drive and the power. A PI controller is used in the feedback circuit to regulate the output voltage.

The output voltage stayed fairly constant in the entire load $(0-500 \mathrm{~W})$ and line $(170-270 \mathrm{~V})$ range. The overall efficiency of the system with $170 \mathrm{~V}$ input with output regulated to $230 \mathrm{~V}$ is $95 \%$ at full load. In Section 4, ZVS in converter 2 was discussed. Figure 12 shows the turn-on and turn-off characteristics of switch S3 in converter 2. It is clear from the Figures that ZVS occurs in converter 2. In Fig. 12 the voltage that swings between positive and negative is the gate voltage. The conduction losses in converter 2 , which amount to $4 \%$, are mainly due to the high $R_{D S(o n)}$ of the MOSFETS used. Switching losses are zero in the output side converter.

In converter 1 since a resistive load is connected at the output, the quadrant of operation is always 2 and 4 . Hence the biasing circuit needs to be connected only for the right leg of converter 1. Simulation results of switch S1 in quadrant 1 are shown in Fig. 13 with and without biasing circuit and the Figure shows a clear reduction of voltage spikes in the waveforms. Figure 14 shows the experimental results of S2 turn-on. It is seen from the Figure that the drain voltage decreases to a lower value before turn-on but does not reach zero voltage. The switching losses are thus 


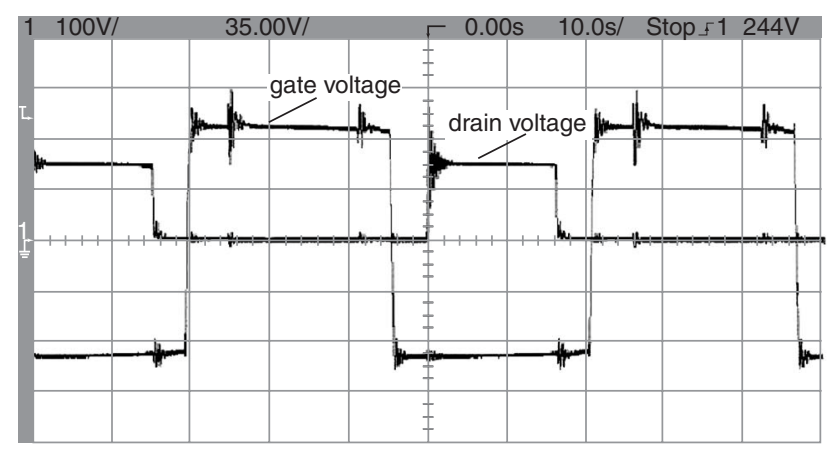

Fig. 12 Observed gate and drain voltage waveform of switch $S 3$ in converter 2 (note ZVS)

voltage across switch $S 1\left(F_{s}=100 \mathrm{kHz}\right)$

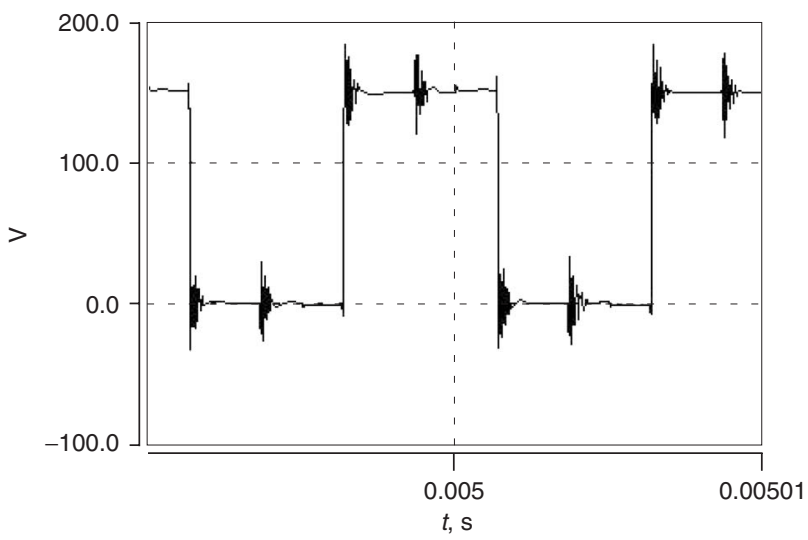

voltage across switch $\mathrm{S} 1$ with biasing circuit $\left(F_{s}=100 \mathrm{kHz}\right)$

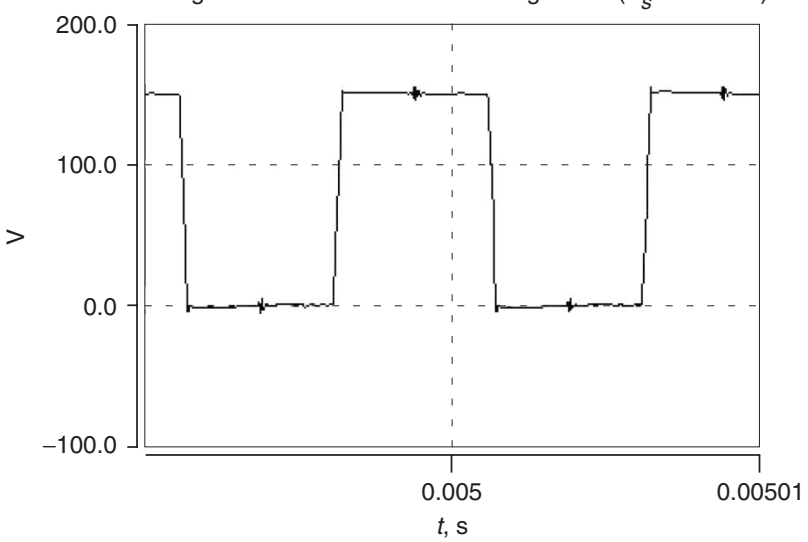

Fig. 13 Simulation results of S1 turn-on with and without biasing circuit

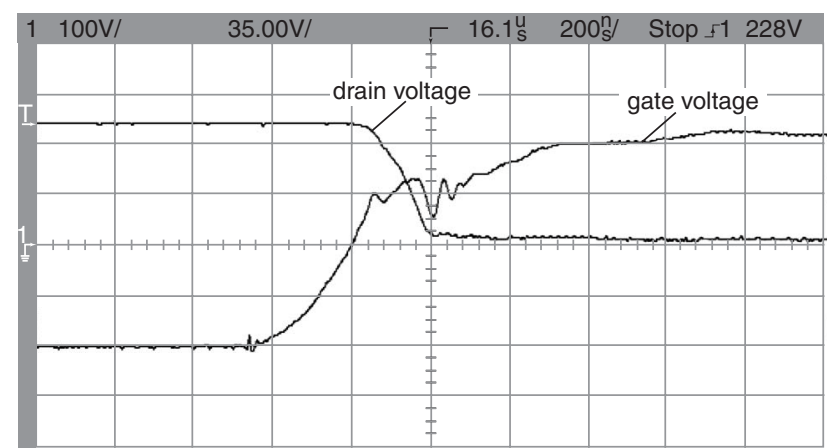

Fig. 14 Observed gate and drain voltage waveform of switch S2 turn-on reduced and the voltage cannot be made zero owing to the absence of a freewheeling diode across the four-quadrant switch. This is still advantageous because in a lossy transition the voltage across the switch goes higher than the bus voltage. The conduction and switching losses are calculated for an input voltage of $170 \mathrm{~V}$ and an output voltage of $230 \mathrm{~V}$, with the AVR delivering $500 \mathrm{~W}$ output. The estimated conduction loss in converter 1 was $10 \mathrm{~W}$, forming the major loss component, and the switching losses were negligible. The total measured losses for both the converters were $25 \mathrm{~W}$.

The experimental waveforms of the AVR for the operating point $V_{\text {in }}=170 \mathrm{~V}, V_{o}=230 \mathrm{~V}$ and $P_{o}=480 \mathrm{~W}$ are shown in Figs. 15 and 16. Figure 15 shows the observed primary voltage and current waveform. The primary voltage envelope is not shown for the sake of clarity. Figure 16 shows the observed load voltage and load current. The waveforms are recorded when the sine voltage is at its maximum and they are shown only for two switching cycles. All the waveforms will have $50 \mathrm{~Hz}$ envelope, which is not shown in the graphs.

\section{Conclusions}

The electronic transformer has been introduced and its principle of operation explained. A symmetric modulation scheme for control of electronic transformer is proposed. The advantages of this scheme, such as unipolar output voltage waveform and ZVS in the output-side converter, are presented. An application of the electronic transformer as an AVR is explained in detail. PWM generation methods for the control scheme are presented. Experimental results of a $500 \mathrm{VA}$ AVR are discussed. This paper explains only one application but the electronic transformer can also find its use in uninterruptible power supplies wherein the isolation transformer at the output side can be replaced by a high-frequency transformer. Finally a novel biasing
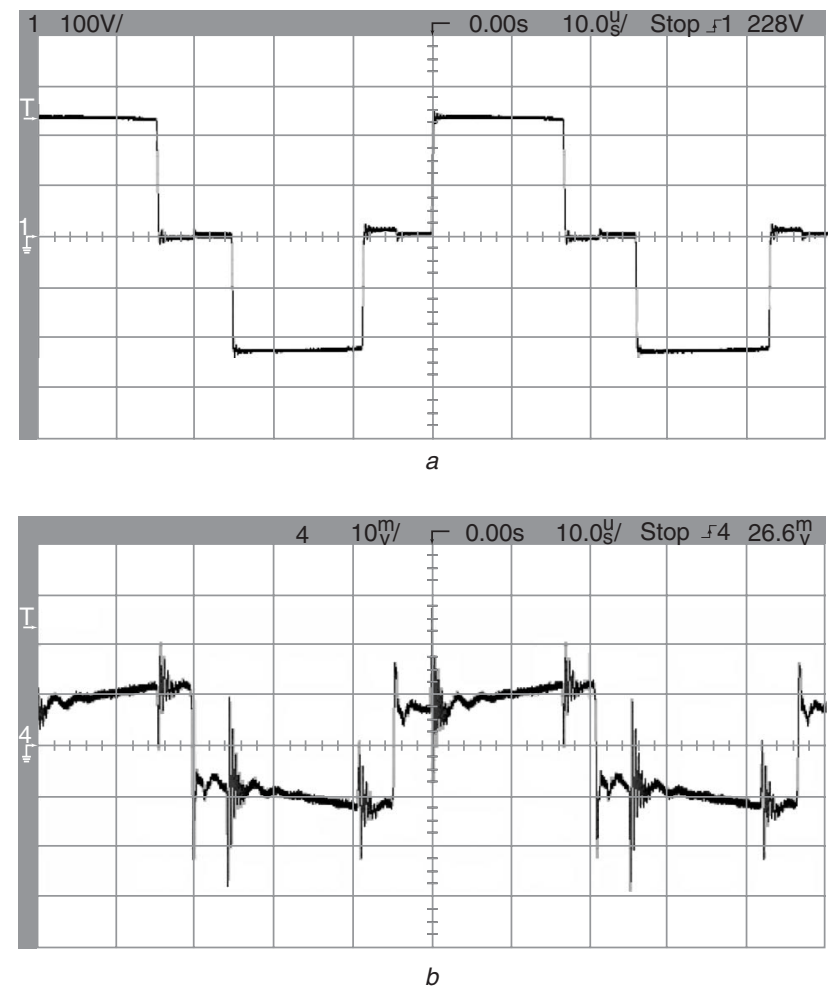

Fig. 15

$a$ Observed transformer primary voltage waveform $b$ Observed transformer primary current waveform 

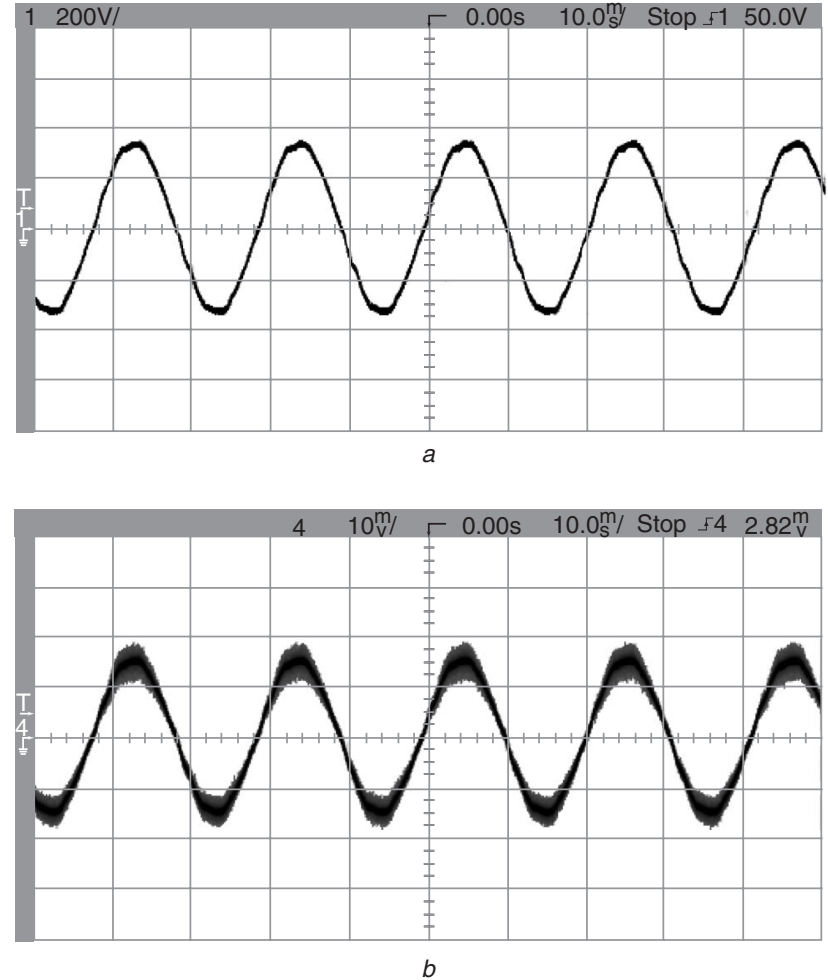

Fig. 16

$a$ Observed output voltage of AVR $V_{0}=230 \mathrm{~V}$

$b$ Observed load current of AVR $I_{L}=2.17 \mathrm{~A}$

Scale: $2 \mathrm{~A} / \mathrm{div}$ circuit is proposed to convert all lossy transitions in converter 1 to lossless transitions and the results are also presented.

\section{References}

1 McMurray, W.: 'Power converter circuits having a high-frequency ink', US Patent 35173000, 23 June 1970

2 Kang, M., Enjeti, P.N., and Pitel, I.J.: 'Analysis and design of electronic transformers for electric power distribution system', IEEE Trans. Power Electron., 1999, 14, (6), pp. 1133-1141

3 Harada, K., Sakamoto, H. and Shoyama, M.: 'Phase-controlled DCAC converter with high-frequency switching, IEEE Trans. Power Electron., 1988, 3, pp. 406-411

4 Hienemann, L., and Mauthe, G.: 'The universal power electronics based distribution transformer - an unified approach'. IEEE PESC2001, Conf. Rec

5 Kwon, B.H., Youm, J.H., and Choi, J.H.: 'Automatic voltage regulator with fast dynamic speed', IEE Proc. Electr. Power Appl., 1999, 146, (2), pp. 201-207

6 Hietpas, S.M., and Naden, M.: 'Automatic voltage regulator using an AC voltage-voltage converter', IEEE Trans. Ind. Appl, 2000, 36, pp. 3-38

7 Hariharan, K.: 'High frequency AC link electronic transformer'. MSc Thesis, Department of Electrical Engineering, Indian Institute of Science, April 2002

8 Enjeti, P.N., and Choi, S.: 'An approach to realize higher power AC controller'. Proc. IEEE APEC Conf., March 1993, pp. 323-327

9 Rajapandian, A., and Ramanarayanan, V.: 'A Constant Frequency Resonant Transition Converter', J. Indian Inst. Sci., 1996, pp. 363-37 